\title{
MICROWAVE TIME DELAY BEAMFORMING USING OPTICS
}

\author{
D Stilwell, M Parent and L Goldberg
}

Naval Research Laboratory

Washington DC 20375-5000

This paper presents a practicable method of driving 2D arrays using time delay waveforms for both transmit and receive. An optical subsystem generates a set of delayed signals which are transferred to the elements by fiber optics. The time delays are generated by creating frequency dependent microwave phase shifts in the optical system. An optical fiber array spatially samples optical wavef ronts and transfers the optical signals to the array where they are heterodyne detected to recreate the microwave signals at the input to TR modules. This process essentially extends the backplane of the antenna back to the control system where element level operations may be performed. All beamforming and nullforming functions may be implemented, e.g. monopulse. The requisite TR modules have no phase shifters and a minimum of logic.

\section{1--INTRODUCTION}

Electronically scanned arrays normally utilize broadband phase shifters to establish the phase gradients across the array to direct beams or synthesize antenna patterns. As a result of approximating time delays by phase shifts only, arrays become quite frequency dependent at high angles of $f$ broadside. The resulting beam direction will change with frequency which precludes directing wideband signals in a single direction. True time delay beam formation has been tried using line lengths and is quite effective for sub-arrays but becomes formidable when applied at the element level. Recently a number of papers have considered the use of fiber optics as a replacement for microwave transmission lines [Koepf]. This paper extends the NRL approach in a manner which creates time delay beamforming without the use of actual switched lengths of transmission line. The next section presents the basic concept of the optical control of array beam formation. Section 3 extends the concept to handle wideband signals, i.e. implements time delay beamforming without the use of variable transmission line lengths. The final section summarizes the paper.

\section{2--FIBER OPTIC FEED CONCEPT}

The present approach fashions an optical miniature of the array in the form of a bundle of fiber optic (FO) cable ends positioned coaxially with the nominal direction of light from a coherent optical system. This bundle of fibers spatially samples light from multiple sources of slightly diverging plane waves. A microwave signal is regenerated at the other end of each fiber upon heterodyne detection.

Figure 1 illustrates the fundamental concepts. The output beams in Figure la provides the input for Figure $1 \mathrm{~b}$. On the left of Figure $1 \mathrm{a}$ is shown a set of three laser diodes injection locked to a master laser. Each of the slave lasers generates a beam of light which, after passing through acousto-optic (AO) devices, mirrors and beamsplitters; falls on the fiber optic array as a plane wave. (The use of laser diodes and injection locking would probably be a poor choice but is used here for illustrative purposes. Electronic stabilization of diode pumped solid state lasers would be a better choice. [Williams]) The central laser path is not modified in either frequency or propagation direction. It provides a reference beam normal to the FO array at frequency $\Omega_{0}$. Each of the other beams, denoted control beams (there can be many but only two are shown for simplicity), passes through an AcoustoOptic Modulator (AOM) and an Acousto-Optic Beam Deflector (AOBD). These AO devices operate on the principle of Bragg scattering but are configured in pairs so as to perform pure frequency shift without beam deflection for the AOM or, pure beam deflection without frequency shift for the AOBD. Therefore, falling on the FO array are a set of plane waves which are coherent but which have microwave offsets in their frequency and which propagate in different directions.

Only two lenses are shown for convenience, and these are used in Figure $1 \mathrm{~b}$ to specify the Fourier transform plane just preceding the FO input plane. It is presumed that collimated light exits the interferometer section of the diagram, Figure 1a. The fibers are assumed to be of the multi-mode type. It should also be noted that there is a bundle of fibers connecting the FO pick- 
up plane with the detector array and that separation may be up to kilometers long with little impact on the circuit performance.

Each fiber intercepts a small fraction of the incident waves energy and transfers it to an element in the array. A photodetector there regenerates a microwave signal by heterodyne detection of the two frequency off set optical signals. Because of the non-zero angle between the optical waves, the microwave signals from different fiber outputs differ in phase. This creates a phase gradient across the array. The array radiates in a direction such that the projection of the wavenumber onto the array exactly matches the phase gradient established by the optical control system. Hence by the process of creating optical beam deflections in one leg of the interferometer of Figure 1, a beam may be directed in any direction from the array.

Figure 2 illustrates a TR module for the FOF system. A fiber end irradiates a photodetector which then feeds a low noise amplifier. This first stage might not be needed for a classical phased array but the low level of the output from the photodetector makes it necessary here. Diode switches select the transmit path or the receive path (shown). On receive, the optical control signals are routed to a mixer. The signals from the control optical system provide the set of local oscillator (LO) signals which will cause the intermediate frequencies (IFs) to combine constructively. Again this is done by creating a phase gradient across the array which is the conjugate of that for the signal to be received. There is also a frequency of fset to create the IF. The set of IF signais can be combined in a number of ways, though a row sum using Lange couplers and a single column sum is adequate. Any phase gradient due to the summing process can be incorporated into the steering vector to have the "point" of summation at the output of the IF bus.

Figure 3 shows experimental data obtained using this design [Tamburrini]. It was taken using a microwave lens collecting the signal from 7 array elements. The on-axis beam was selected but the phase gradient across the array was varied linearly by frequency sweeping the signal into the AOBD in one of the paths. The resulting pattern is an array factor pattern. The real space from such an array would be constrained to the region $\pm \pi$ phase shift between elements. Because of stray coupling in the measuring circuit the magnitudes do not render faithfully, but the fact that null depths are quite deep indicates that accurate control existed on the 7 distinct amplitudes and phases, i.e. less than a degree rms phase error.

In its simplest conceptual form the previous section describes an optical system dedicated to creating two point sources of laser light in a Fourier transform plane (denoted the $\mathrm{K}$-space plane) immediately preceding the fiber optic pickup array. The sources, e.g. those denoted by 0 and
1 in Figure 1, are derived ultimately from a common source of light with a frequency of fset in one of them obtained by external modulation. They also differ in position due to the beam deflector which causes a divergence between the outgoing plane waves. The vector connecting the two points in the $\mathrm{K}$-space plane is proportional to the phase gradient between fibers and thereby determines the gradient at the array of microwave elements. The two sources give rise to plane waves describable in simple phasor notation which on mixing at the detectors yields a single microwave phasor involving the difference wavenumber and difference frequency between the optical quantities. The argument of that phasor is a linear function of array element position, the coefficient being the phase gradient. When three optical beams are used as indicated in Figure 1, one of the possible Kspace diagrams would be that indicated in Figure 4. This indicates that the antenna pattern is to have two major lobes. This would constitute pattern synthesis if the two lobes are at the same frequency, but they can be any two frequencies within a given octave. The appropriate aperture function would define the pattern, the $\mathrm{K}$-space directions only indicate the direction of the main beam.

This architecture has a number of distinct advantages. One of these would be that the lasers used can be very quiet since direct modulation at the microwave frequency is not required and lasers with low relative intensity noise at microwave frequencies can be chosen. Another is that the control system can be quite remote from the antenna since the loss in fibers is small. Along with the intrinsic advantages of weight, size, and flexibility; this architecture represents a powerful realization for beam synthesis and adaptivity applicable to two dimensional arrays.

\section{3--WIDEBAND BEAMFORMING}

To launch a band of microwave frequencies in some given direction from the antenna, a different source separation is required for each frequency in the $\mathrm{K}$-space diagram. This can be accomplished by the use of a third source term and a frequency dependent partitioning of the signal. Figure 5 illustrates the concept. It shows the incoming signal being divided between opposite slope discriminators. The outputs are then fed to two control beams in the optical system, at the AOM inputs. This introduces weighting onto the $\mathrm{K}$-space sources; relative weight $>1$ for the reference beam, weight $\alpha$ for one of the controlled beams and weight $1-\alpha$ for the second controlled beam. Figure 5 also shows the appropriate $\mathrm{K}$-space diagram, now consisting of the reference source at frequency $\Omega_{0}$ on-axis and two controlled sources each at frequency $\Omega$. The parameter $\alpha$ partitions the source strength in amplitude all to source 1 at one extreme of the discriminator span and all to 
source 2 at the other extreme. These extreme frequencies are controlled by the beam deflectors to be directed in some given direction from the microwave array. By this procedure any frequency within the span of the discriminators will be directed in the same direction from the microwave array.

The key conceptual ingredient manifest in this process is the introduction of a phase shift between fiber inputs (and consequently antenna elements) which is a linear function of frequency. By the shift invariance property of Fourier transforms the partial derivative of the argument of the resulting phasor with respect to frequency is a time delay. This time delay is also a linear function of the array position and therefore describes a pulse sweeping across the array.

The introduction of 2 sources at frequency $\Omega$ in the source diagram will result in a 2-beam interference pattern which would in turn introduce an amplitude variation over the array. This would modify the resulting antenna pattern. This is true but it is a necessary feature of a broadband signal radiating from an extended structure. It is not possible to sum a set of phasors depending on $k, \omega$ and position, $x_{i}$, without introducing an amplitude variation.

To receive a pulse from some given direction with the antenna, a range of phase gradients (projected wavenumbers) must be synthesized so that the mixers in the TR modules will shift the spectrum to IF and provide time delays across the array. This can be done in the optical subsystem by use of the reference beam and a single control beam channel. The span of wavenumbers at a fixed frequency, $\Omega_{l o}$, required in the $K$-space diagram can be obtained by optically defocusing the source for the control beam. This will yield in one dimension only, a quadratic phase factor across the fiber optic pickup. This will then effect a range of phase gradients across the aperture. Each component of the incoming wideband signal will coherently interact with a small range of the phase gradient span. The LO signal can be viewed as a set of orthogonal signal components, each of which will select only a small range of frequencies from the received signal. The entire set will, however, regeneiate the wideband signal.

\section{4--SUMMARY AND CONCLUSIONS}

This paper has presented a method of beam control and synthesis appropriate for antenna arrays. It performs the beam forming functions in an optical miniature of the intended array by creating point sources of coherent light to provide the phasors required. The lateral properties of the optical plane waves sampled by a fiber optic array are controlled by AOBDs. All beam and waveform operations associated with radars can be implemented with the architecture; pulse compression, intra-pulse beam steering, monopulse, multiple independent beams, wideband nulls, etc. The requisite TR module requires a minimum of components, no phase shifter and only 2 bits of logic.
Of particular importance is the ability to nondispersively direct wideband energy in any given direction from broadside to the array. This feature derives from the creation of frequency dependent phase shif ts in the control optical system which uses frequency discriminators. The resulting time delay beamforming is accomplished without the use of actual RF transmission line lengths and can be centinuously varied.

References:

[Koepf] Koepf, G.A., Proc. SPIE, 1984, 477 (Washington, D.C.)

[Tamburrini] M. Tamburrini, M. Parent, L. Goldberg and D. Stilwell, Electronics Letters, v 19, pp 680-681, 1987

[Williams] K.J. Williams, L. Goldberg, R.D. Esman, M. Dagenais, and J.F. Weller; Electronics Letters, v. 25, pp $1242-1243$ 


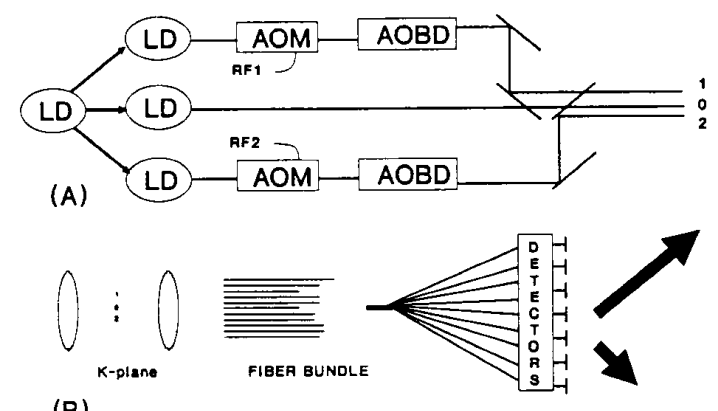

(B)

Figure 1--Fiber optic feed concept.

(A) Interferometer forming plane waves.

(B) Fiber pickup and antenna array.

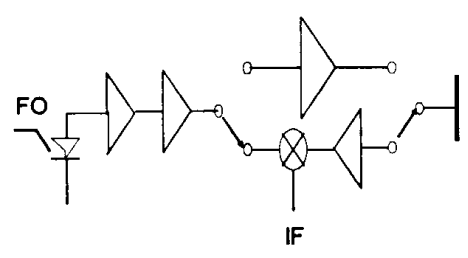

Figure 2--TR module.

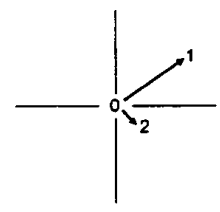

Figure 4--K-space diagram for floure

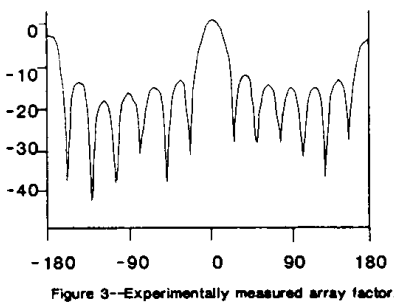

Figure 3-Experimentally mesourod erray iector.

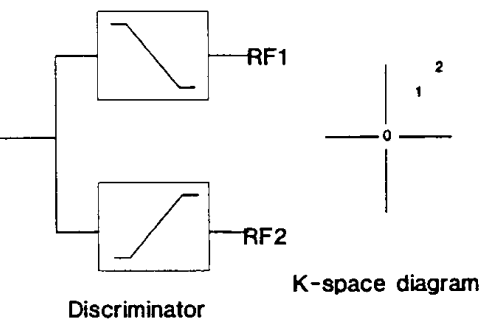

Figure 5--Wideband signal conditioning. 